This is an author produced version of a paper published in Proc. of IEEE/ION Position Location and Navigation Symposium (PLANS), 2012.

This paper has been peer-reviewed but does not include the final publisher proofcorrections or proceedings pagination.

(C) 2012 IEEE. Personal use of this material is permitted. Permission from IEEE must be obtained for all other uses, in any current or future media, including reprinting/republishing this material for advertising or promotional purposes, creating new collective works, for resale or redistribution to servers or lists, or reuse of any copyrighted component of this work in other works.

Citation for the published paper:

Rantakokko, J., E. Emilsson, P. Strömbäck, J.Rydell, Scenario-based evaluations of high-accuracy personal positioning systems

Proc. of IEEE/ION Position Location and Navigation Symposium (PLANS), 2012.

Access to the published version may require subscription.

Published with permission from: IEEE 


\title{
Scenario-based evaluations of high-accuracy personal positioning systems
}

\author{
J. Rantakokko ${ }^{1,2}$, E. Emilsson ${ }^{1}$, P. Strömbäck ${ }^{1}$ and J. Rydell ${ }^{1}$ \\ ${ }^{1}$ Swedish Defence Research Agency (FOI), Linköping, Sweden \\ ${ }^{2}$ Signal Processing Lab, ACCESS Linnaeus Centre, KTH Royal Institute of Technology, Stockholm, Sweden \\ \{jounir,eriemi,pstrom,joaryd\}@foi.se
}

\begin{abstract}
Foot-mounted inertial sensors combined with GPSreceivers, magnetometers, and barometric pressure sensors have shown great potential in providing high-accuracy positioning systems for first responder and military applications. Several factors, including the type of movement, surface, and the shape of the trajectory, can strongly influence the performance of footmounted inertial navigation systems. There is a need for realistic scenario-based evaluations as a complement to the controlled environment tests that have been published in the literature. In this work we evaluate the performance of a foot-mounted inertial navigation system using three-axis accelerometers, gyroscopes and magnetometers during realistic scenario-based measurements. The position accuracy is evaluated by using a camera-based reference system which positions itself towards visual markers placed at pre-surveyed positions, using a slightly modified version of the ARToolKitPlus software. Maximum position errors of 2.5 to 5.5 meters were obtained during four separate high-tempo building clearing operations that lasted approximately three and a half minutes each. Further improvements in accuracy, as well as improved robustness towards different movement patterns, can be achieved by implementing an adaptive stand-still detection algorithm.
\end{abstract}

\section{INTRODUCTION}

There is a consensus in the research community that the viable approach for providing, in the near-term, an accurate, robust positioning system with seamless outdoor and indoor coverage is to utilize a multi-sensor fusion approach combining different positioning sensors. GPS receivers, inertial sensors, radio-based ranging, magnetometers, barometric altimeters, ultrasonic sensors, Doppler radars and imaging sensors are all of interest for inclusion in such a system (see e.g. [1]). The user requirements on size, weight, cost and power efficiency (SWaP-C) that have been described by first responders and military personnel are stringent. Also, the operational environments will be diverse, and the inclusion of pre-installed infrastructure or a priori information (e.g. building layouts or image information) is mostly considered unfeasible.

Experiments using foot-mounted inertial navigation systems (INS) supported by zero-velocity detection and updates was published already in 2005 [2]. Since then, several research groups have demonstrated high accuracies with experimental foot-mounted inertial navigation systems in

Manuscript revised on 7/5/2012. controlled environment tests, highlighting the possibilities with the technology in safety and security applications (see for instance [3] and [4]). The key for achieving an accurate footmounted inertial navigation system is the ability to reliably detect a stance phase using data from the foot-mounted inertial measurement unit (IMU). Prior work within this area has often been based on applying empirically determined thresholds on accelerometer and/or gyro readings, see e.g. [5]. This approach normally performs well during walking; however, more robust stand-still detection algorithms are desired that can handle all relevant movements, including sprinting, jogging, sidestepping, ascending and descending stairs, and crawling [4].

There is still a lack of performance evaluations conducted during more realistic conditions in the open literature. Several factors can strongly influence the performance of foot-mounted inertial navigation systems, such as the type of movement and surface (which both can affect the stand-still detection performance), the shape of the trajectory (which can hide heading errors), local magnetic disturbances, etc. Also, the number of units and their distances, as well as the radio channel properties, are of interest in examinations of the performance of cooperative positioning strategies [1]. Hence, as a complement, there is an apparent need for scenario-based evaluations, both for vendors in their work with improving the system performance as well as for the end-user community in providing knowledge needed for upcoming requirements definition and procurement phases.

Furthermore, automatic reference systems providing a means for evaluating the position accuracy during the exercise are desired, when evaluating for instance the influence of the trajectory or type of movement on the positioning error [6]. Merely evaluating the position error when returning to the start point may provide deceptive results.

As a motivating example, the new generation of inertial measurement units can fulfill demanding user requirements concerning size, weight and power efficiency. For instance, the Xsens MTw IMU weighs 27 grams including battery and wireless sensor data transfer capability, and it is well suited for boot-mounting [7]. The potential market for high-accuracy positioning systems is large, targeting both the first responder and military communities. Several companies have indicated plans for releasing products during 2012. Cost could still prove prohibitive for achieving high market penetration but the unit price is expected to go down as the market takes off. 


\section{FOOT-MOUNTED INERTIAL NAVIGATION SYSTEM}

The principle for inertial navigation systems can be briefly described as follows. The three-axis gyros provide angular velocities which are integrated to angles that describe the inertial sensors orientation relative a chosen coordinate system. When the orientation of the sensor is calculated, then the measured acceleration can be expressed in terms of components in a local coordinate system (e.g. north, east and down, NED) and the measured acceleration due to gravity can be compensated for. The gravity-compensated measured acceleration is then integrated twice, providing first the velocity and thereafter the position.

At each step, the fact that the foot is at stand-still for a short period of time is used as a pseudo-measurement of zerovelocity in the filter. This approach has proven to efficiently limit the error growth of the navigation solution. The navigation algorithm is based on a 9-state Extended Kalman Filter, which estimate the 3D-position, velocity and orientation of the IMU. A coarse estimate of the gyro bias is obtained by using the mean value of the measured angular velocity during an initial period of stand-still.

A simple threshold-based method for detecting foot standstill was utilized in this work. If the root-mean-square value of the angular velocity, during a period of $0.05 \mathrm{~s}$ (five samples using $100 \mathrm{~Hz}$ sampling rate), is below a threshold of $0.3 \mathrm{rad} / \mathrm{s}$ a zero-velocity update is performed for each of these five samples. The threshold level was determined empirically and it has shown to work fairly well in many of our previous tests. The optimal threshold level can however be affected by several factors, such as the motion of the person (e.g. walking vs. running), weight carried, surface (e.g. asphalt, sand, mud, gravel, indoors) and shoe type, and if the person moves in stairs (up- or downwards). Adaptive stand-still detection algorithms which automatically adjust thresholds towards the present conditions, for instance movement speed, are expected to further improve the navigation accuracy [4].

When using the magnetometer it is crucial that local disturbances of the magnetic field are not allowed to affect the results. The magnetic field is often significantly disturbed in indoor environments, by electronic equipment, metal objects, electrical wires, etc. Also, the equipment worn by for instance a firefighter can cause magnetic disturbances which can make it difficult to use magnetometers without proper filtering. An innovation filter approach was taken herein to reduce the effects of locally generated magnetic fields. Also, magnetometer data is only used when the foot is at stand-still.

\section{REFERENCE SYSTEMS}

Future high-accuracy positioning systems strive at providing meter-level accuracy during the extent of typical operations. In a firefighter scenario this normally requires the system to operate indoors for up to 20 or 30 minutes, while other applications may experience significantly longer periods in indoor environments. Any reference system used for evaluating the performance of such systems must provide even higher accuracy, preferably a factor 5 higher. Otherwise, long duration tests must be supported in order to evaluate the system performance thoroughly.

\section{A. Different possibilities for providing reference positions}

In the evaluation phase accurate reference positions can be obtained in a number of different ways, e.g.:

- The person returns to the starting position enabling an easy means of estimating position error.

- The person passes over, and possibly stops at, a number of pre-surveyed reference positions.

- Using a so-called total station which uses laser to measure distance, and accurately estimates bearing, to a prism placed on the person (e.g. helmet).

- The person is equipped with a camera which positions itself accurately towards pre-installed visual markers at known positions.

- Using pre-installed systems covering a certain area (normally a room or building), using RF-based technology (e.g. Ultra-Wideband (UWB) transceivers), cameras or acoustic sensors.

The first two approaches are easy to employ and do not require any extra equipment. However, relying on the first approach may provide inaccurate results, e.g. since some trajectories reduce the effects of the often dominating heading errors (e.g. moving multiple times in symmetric closed-loops). The second approach requires the test person to restrict his motion, which may lead to different movement patterns than is the case in real scenarios. Total stations can provide extremely accurate reference positions, but they are expensive and require constant line-of-sight to the prism. The latter two approaches provide an opportunity to perform scenario-based exercises.

These techniques can also be combined with using building layouts and plotting the estimated positions on the map. This quickly enables an intuitive feeling for the accuracy of the system. The foot-mounted INS must then be initialized (heading and position) so that it can be associated correctly with the building map.

\section{B. Camera-based reference system using visual markers}

General camera-based positioning is a difficult problem, requiring tracking of a large number of landmarks. By installing markers at known positions, an easier problem is obtained. In marker-based positioning, the localization is performed in two steps:

- An approximate global position is found by determining which (if any) marker is visible. Since the global position of each marker is known, a simple table lookup provides the camera position with an accuracy of a few meters (assuming that the markers are too small to be detected and identified at larger distances).

- Once a marker is found, more precise local positioning is performed by inspection of its appearance in the image. Provided that the camera is reasonably well positioned relative to the marker (with respect to distance and angles), an estimate of the camera position relative to the marker can be obtained. 
A slightly modified version of the ARToolKitPlus toolbox is used herein to estimate the cameras position based on visual markers [6]. It provides accurate (decimeter level) positions at distances of up to three meters from the respective markers, during realistic lighting conditions and movements.

The initial position and orientation of the foot-mounted system is unknown. The estimated trajectory is in these analyses aligned to the coordinate system of the reference system. This can be performed by designing the trajectory such that it begins with a simple motion (e.g., walking straight ahead for a few meters). A number of reference markers are located along this first part of the trajectory, and the initial position and orientation of the foot-mounted system is found by minimizing the difference between the first parts of their estimated trajectories. The time shift between the two systems is also found by minimizing this difference. The reference system does not impose any requirements on the motion except during this short alignment procedure.

\section{DESCRIPTION OF MEASUREMENTS}

A two-story building, consisting of two apartments on each floor, was searched during the exercise. A building layout and photograph of the building interior (including visual markers) can be seen in Fig. 1. Four separate tests were performed (see Fig. 2). In the first two tests one soldier performed the search, while two soldiers were involved during the third and fourth tests. The task given to the soldiers was to search all four apartments, and act as the search was actually performed by a group of soldiers. However, in the first test, the soldier did not search the rightmost apartment on the ground floor. The soldier started outside the building and returned to the same position after each search. The start position was a few decimeters below the building in height. The height difference between the floors was approximately 2.70 meters.

A MicroStrain 3DM-GX3-25 IMU was placed on the soldier's boot. It is equipped with accelerometer, gyro and magnetometer triads. The dynamic range of the accelerometers and gyros are $16 \mathrm{~g}$ and $1200 \% \mathrm{sec}$, respectively. No barometer was available during the scenario-based tests. An Xsens MTw IMU was also used but hardware problems made the results unreliable.

The soldier was equipped with a separate camera which was placed on a back-pack or at the helmet. The camera provided a means to position the soldier towards pre-installed visual markers during these tests. In test 4 , the camera was placed on the helmet at a height of about 2 meters above ground while the markers were placed less than 1.5 meters above the floor. In the first three tests the camera was mounted on the shoulder or backpack, at a somewhat lower height, providing more reference position measurements.

\section{RESUlts}

In Fig. 3, the estimated trajectories are compared for the foot-mounted INS (without magnetometer) and a visual-inertial navigation system using a stereo-camera that is co-located with an Xsens MTi IMU [9]. The test subject walked around the building and both systems display an error of only a few decimeters. The difference in trajectories is mainly due to the fact that the IMU is placed on the person's right foot while the camera is hand-carried. During walking the foot-mounted INS has no problems detecting the periods of stand-still of the foot, and high accuracies are generally obtained. A crude estimated map obtained from the identified landmarks from the camerabased system is also shown (gray point clouds) in Fig. 3 [9].

The difference in position estimates (horizontal positions) between the reference system and the foot-mounted INS are shown in Fig. 4 to Fig. 7 for the four scenario-based tests. The results are shown with (blue) and without (red) the use of the magnetometer data. Note that the vertical spread in distance is caused by the varying distance between the foot and the camera when the person passes a marker. The evaluation is performed during periods where zero-velocity was detected, where the horizontal difference in position due to the camera and foopositions are smallest. The results indicate how the error increases during the course of the exercise. The first conclusion is that the position error does not necessarily increase with time/distance - in several cases the error is reduced during the latter part of the test.

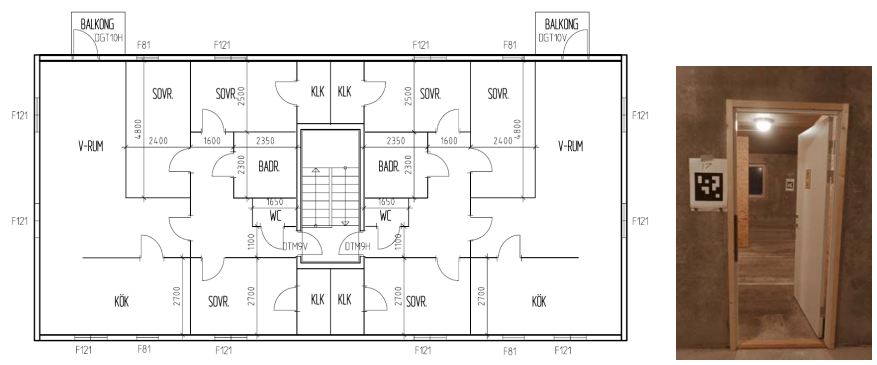

Figure 1: $2^{\text {nd }}$ floor building layout (left) and picture of building interior and visual markers (right).

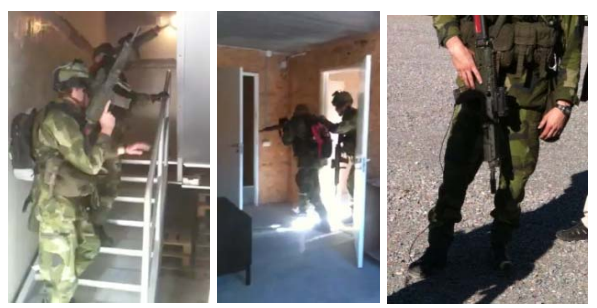

Figure 2: Pictures taken during exercise (left and middle). Soldier equipped with foot-mounted IMU and cable to processing unit (right).

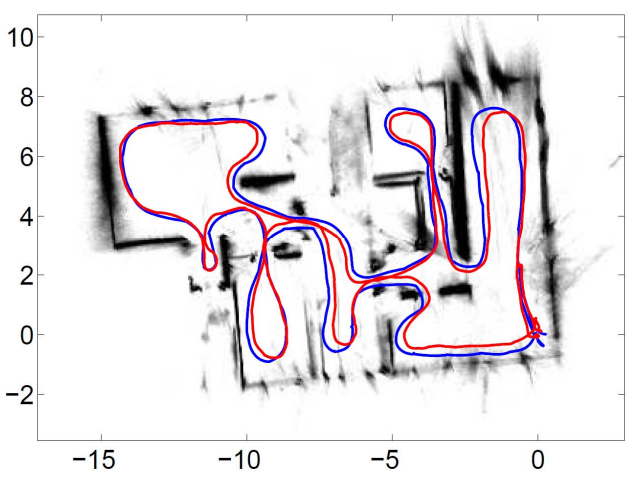

Figure 3: Estimated trajectories for a foot-mounted INS (blue) and a visualinertial navigation system (red) 


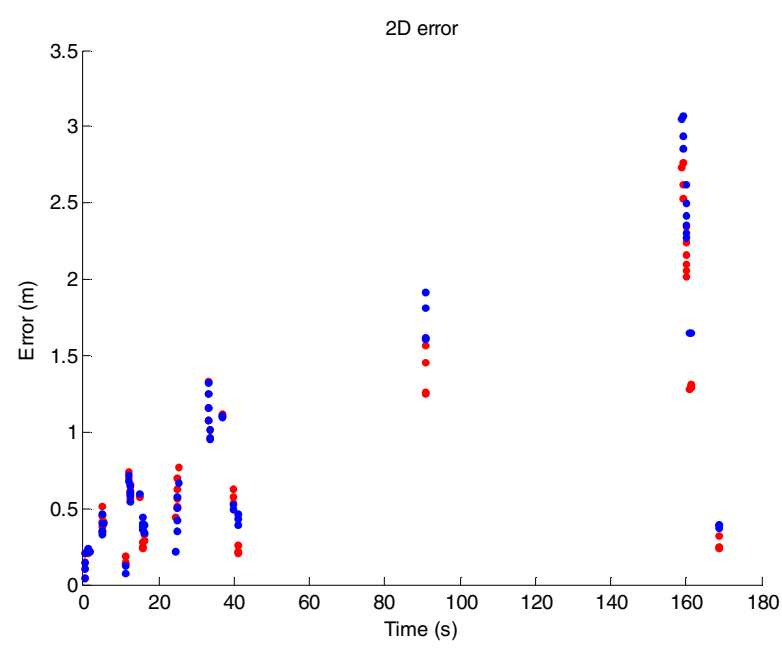

Figure 4: Test 1. Difference in position between camera-based reference system and foot-mounted INS, with (blue) and without (red) use of magnetometer.

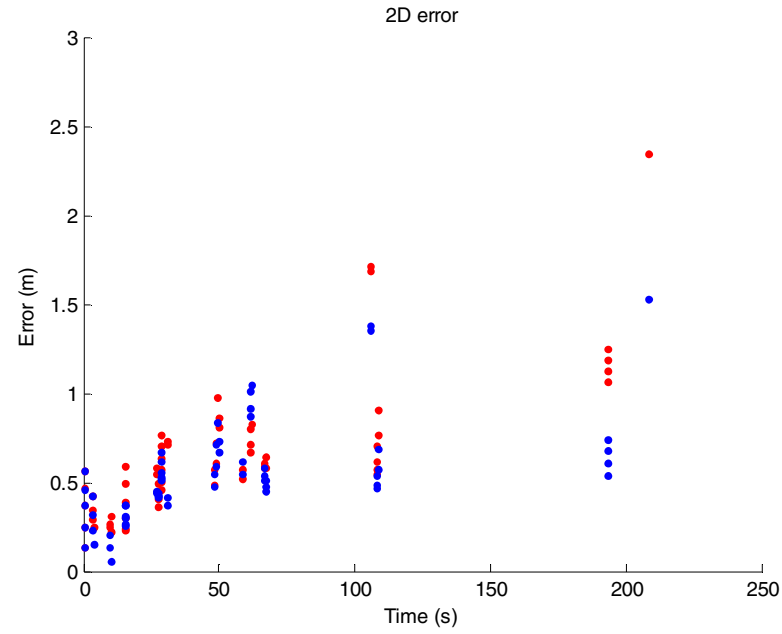

Figure 5: Test 2. Difference in position between camera-based reference system and foot-mounted INS, with (blue) and without (red) use of magnetometer.

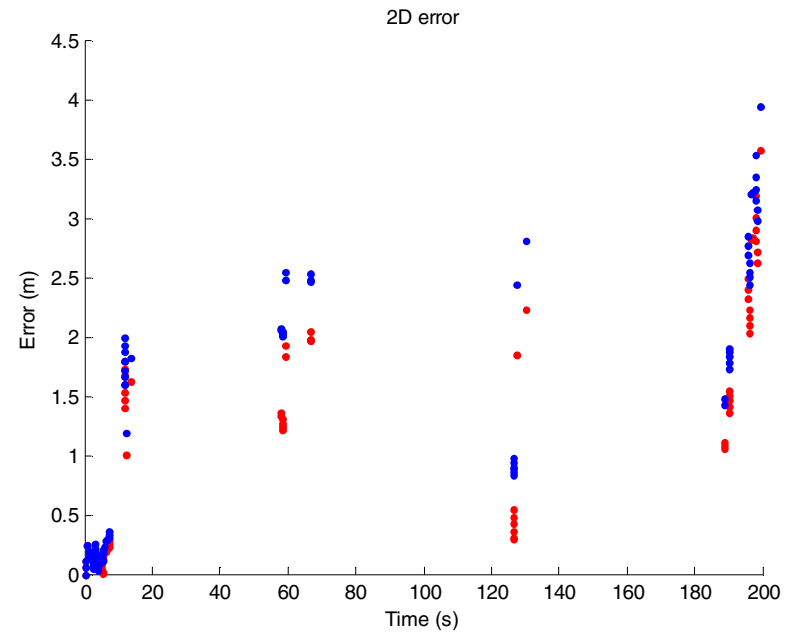

Figure 6: Test 3. Difference in position between camera-based reference system and foot-mounted INS, with (blue) and without (red) use of magnetometer.

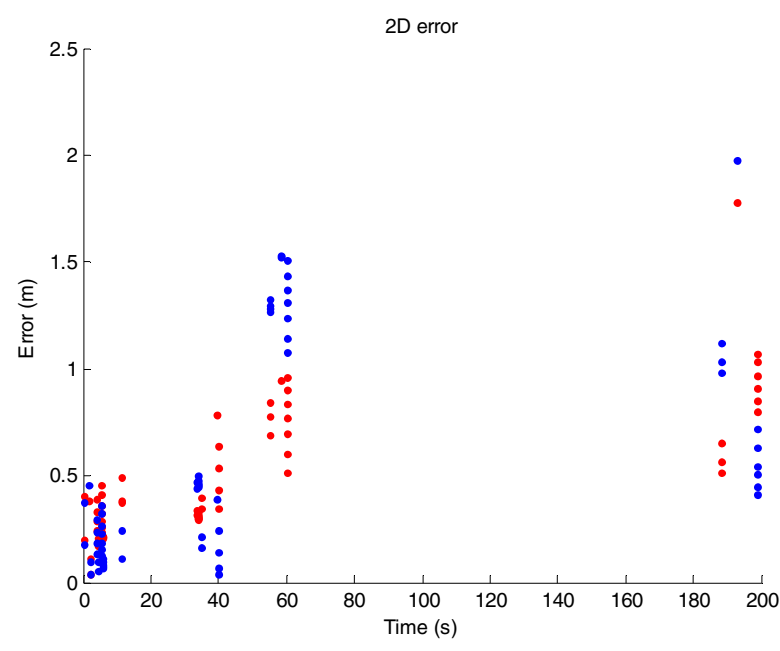

Figure 7: Test 4. Difference in position between camera-based reference system and foot-mounted INS, with (blue) and without (red) use of magnetometer.

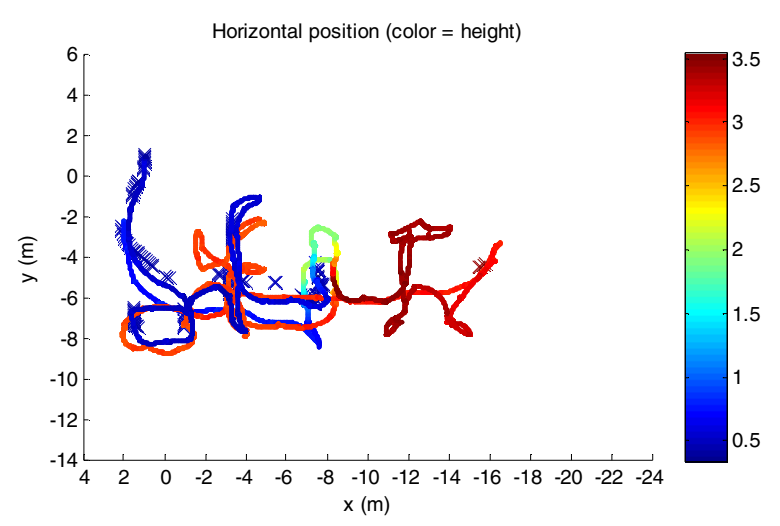

Figure 8: Test 1 . Trajectory for foot-mounted INS (color-coded with height estimate) and reference system positions (' $x$ '). No magnetometer was used.

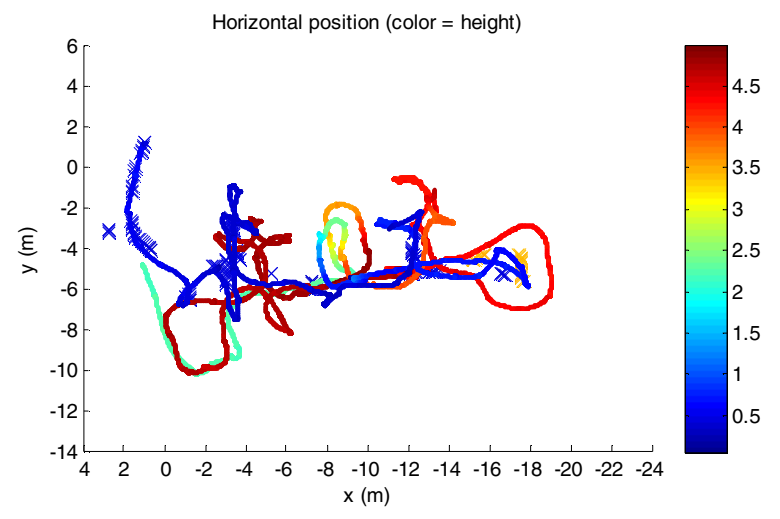

Figure 9: Test 2. Trajectory for foot-mounted INS (color-coded with height estimate) and reference system positions (' $x$ '). No magnetometer was used. 


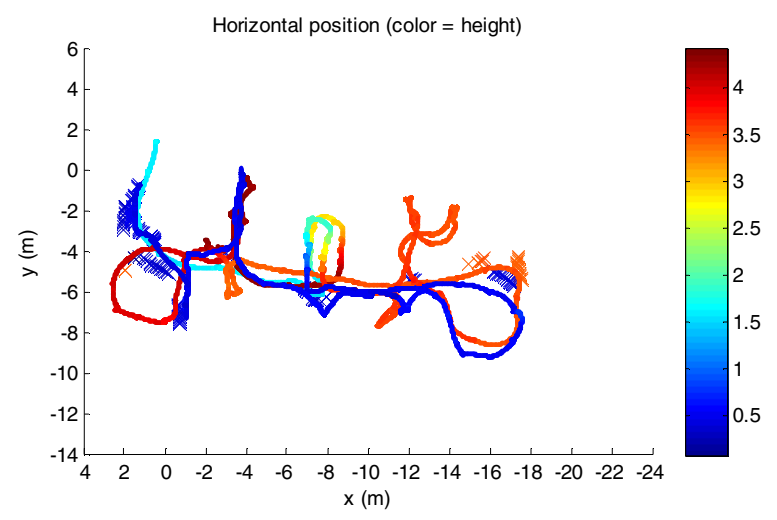

Figure 10: Test 3. Trajectory for foot-mounted INS (color-coded with height estimate) and reference system positions (' $x$ '). No magnetometer was used.

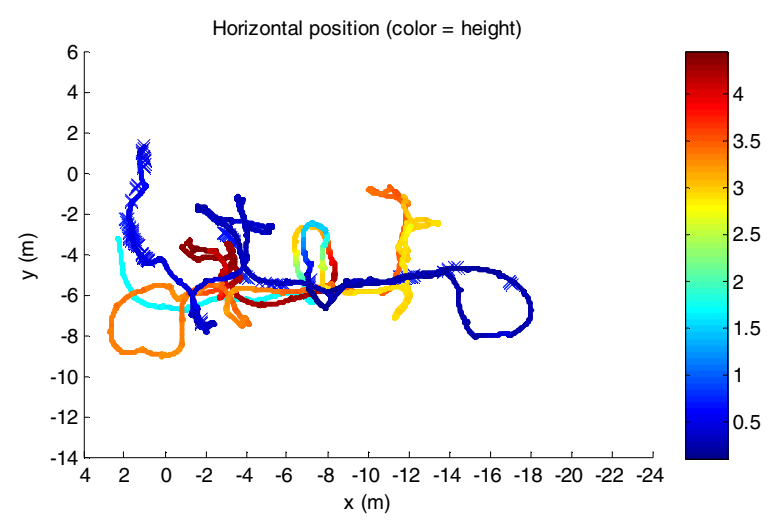

Figure 11: Test 4. Trajectory for foot-mounted INS (color-coded with height estimate) and reference system positions (' $x$ '). No magnetometer was used.

At the end of the four measurements the position error is estimated to be between 1 to 3 meters. The maximum error during the tests (evaluated at positions where visual markers where detected) was below 3.5 meters.

Magnetometers have previously proven valuable in outdoor operations, particularly in GNSS-denied environments. Magnetometers can improve the accuracy also in indoor scenarios [10]. Improved results using magnetometer data can be seen in test 2 in Fig. 5. However, the filter needs further improvement since the magnetometer also increases the error in some cases. The equipment worn by a first responder or soldier may cause additional magnetic disturbances, as manifested in these measurements, and careful design of the navigation filter is required. Note that, in these tests no calibration of the magnetometer was performed after it was placed on the boot.

In Fig. 8 to 11 the estimated horizontal trajectories for the foot-mounted INS are shown. The trajectories are color-coded so that the estimated height is also available. The reference position measurements are also included, marked by ' $x$ ', where orange or red markings in Fig. 9 and 10 indicate reference measurements from the second floor. The point $(0,0)$ in the local coordinate system is set to the position of the first detected visual marker (which is the same in all four tests).

The estimated heights are shown in Fig. 12 to 15 . The height errors in the end of the measurements are below two meters for these relatively high-dynamic scenarios. The abrupt height changes at the second floor in Test 2 and 4 are likely caused by the soldiers kicking in doors during the exercise, or by short periods without zero-velocity updates. The height estimate is however very sensitive to the choice of stand-still detection threshold values used. In indoor operations it is possible to apply floor-pinning approaches in order to improve the height accuracy.

\section{DISCUSSION}

The main interest in this work lies in the evaluation process, where scenario-based evaluations provide an important tool. Evaluations of performance in realistic scenarios with typical movements showed that fairly high accuracies can be obtained also during realistic operations. However, the accuracy must be improved further in order to fulfill user requirements.

The use of external reference systems proved useful in the evaluations. For example, the horizontal position error when the person returned to the original starting position (located outside the building) for tests 1 and 4 were 0.6 and 1.7 meters respectively, which is smaller than obtained with the reference system (Fig. 4 and 7) during the tests. A somewhat higher density of reference positions is desired than was available; however, the process of surveying the marker positions is costly and the number of markers should be kept to a minimum. Careful placement of markers is preferred instead, where marker positions are discussed together with end-users in order to improve the chances of them being detected.

Although a relatively high accuracy is achieved with the experimental system, compared to what is expected for existing systems based on hip- or back-mounted inertial sensors, more accurate foot-mounted INS are likely available. (Test results for systems closer to prototypes or products are often restricted). For instance, we have used the MicroStrain 3DMGX3-25 IMU, which was released a couple of years ago. Although it has provided fairly accurate results, higher quality gyros are currently available with similar form factors provided by e.g. InterSense NavChip or Sensonor's STIM300 (which uses a butterfly gyro originally developed by IMEGO). The major error source is the heading drift, which is strongly affected by the quality of the gyro sensors.

\section{CONCLUSIONS AND FUTURE WORK}

Maximum horizontal position errors of under 3.5 meters were obtained during four separate high-tempo building clearing operations that lasted almost three and a half minutes each. Magnetometers can improve the performance in indoor environments but continued work is required in order to avoid situations where the magnetometer reduces the accuracy. 


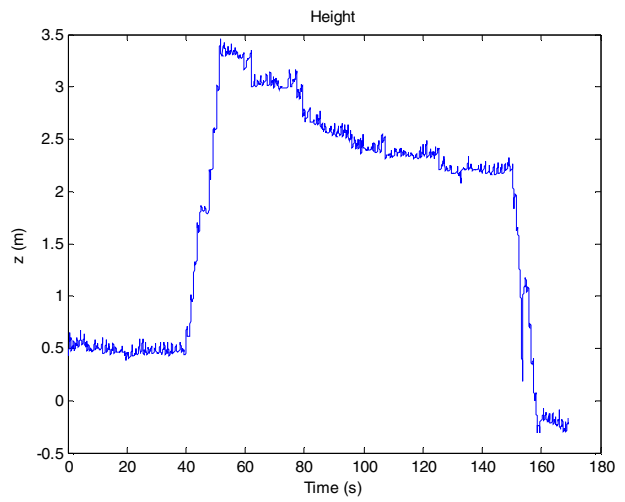

Figure 12: Test 1. Estimated height vs. time.

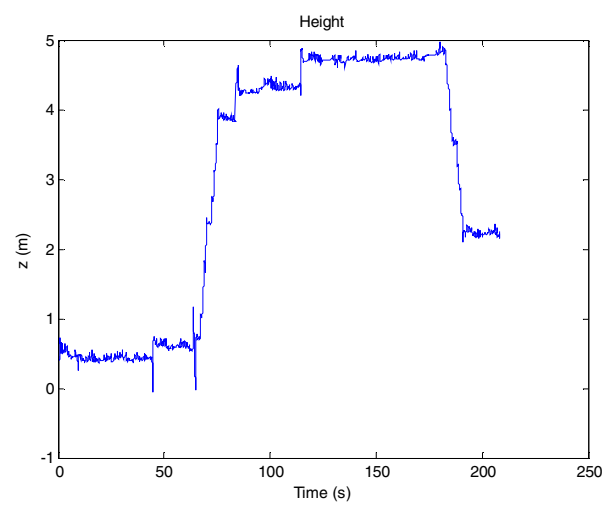

Figure 13: Test 2. Estimated height vs. time.

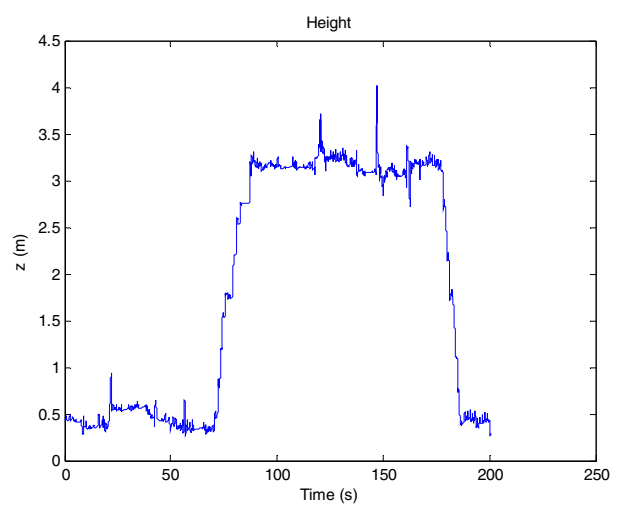

Figure 14: Test 3. Estimated height vs. time.

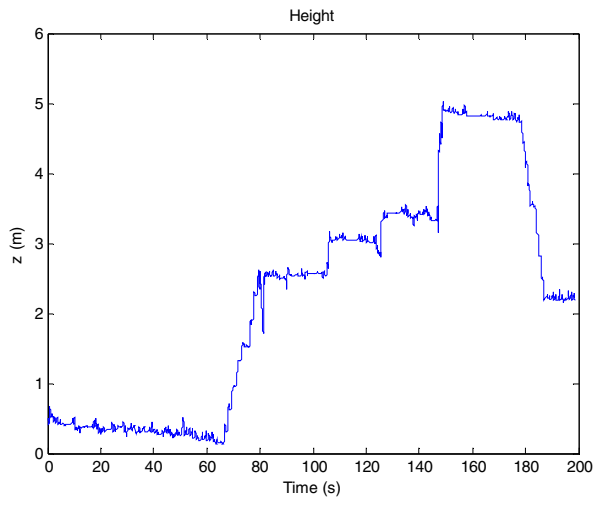

Figure 15: Test 4. Estimated height vs. time.
Proper magnetometer calibration is important. If the accuracy of the inertial navigation system is further enhanced, then the use of magnetometers indoors becomes less important and it also becomes more difficult to design and tune the innovation filter properly.

Returning to the starting position at the end of the measurement and using this position error as the sole performance metric is unsatisfactory. The use of external reference systems, or pre-surveyed reference points which the person moves over during the test, are required in order to obtain realistic performance evaluations.

Several ways to improve accuracy are being explored:

- $\quad$ Adaptive algorithms for improved stand-still detection during multitude of movement patterns.

- Classification of irregular movements such as crawling and elevators, and improved positioning strategies for these.

- Evaluation of performance using dual IMU's, including different placements for these.

- Integration of camera-based SLAM [9] and footmounted INS.

- Combining accurate UWB-ranging with foot-mounted IMU's, thereby enabling the use of (opportunistic) cooperative positioning strategies for long-term operations [1].

\section{REFERENCES}

[1] J. Rantakokko, P. Strömbäck, J. Rydell, P. Händel, J. Callmer, D. Törnqvist, F. Gustafsson, M. Jobs, and M. Grudén, "Accurate and reliable soldier and first responder indoor positioning: multi-sensor systems and cooperative localization," IEEE Wireless Communications Magazine, April 2011.

[2] E. Foxlin, "Pedestrian tracking with shoe-mountedinertial sensors." IEEE Computer Graphics andApplications, November/December 2005 (vol 25, no.6) pp. 38-46.

[3] S. Wan and E. Foxlin, "Improved pedestrian navigation based on driftreduced MEMS IMU chip," Proceedings of ION International Technical Meeting (ITM), San Diego, CA, USA, January 2010.

[4] U. Walder and T. Bernoulli, "Context-adaptive algorithms to improve indoor positioning with inertial sensors," Proceedings of Indoor Positioning and Indoor Navigation (IPIN), Zurich, Switzerland, September 2009.

[5] I. Skoog, P. Händel, J.-O. Nilsson, and J. Rantakokko "Zero-velocity detection in pedestrian navigation systems - an algorithm evaluation," IEEE Transactions on Biomechanical Engineering, vol. 57, no. 11, November 2010.

[6] J. Rydell and E. Emilsson, "(Positioning evaluation)2," Proceedings of Indoor Positioning and Indoor Navigation (IPIN), Guimaraes, Portugal, September 2010.

[7] www.xsens.com

[8] P. Strömbäck, J. Rantakokko, S.-L. Wirkander, M. Alexandersson, K. Fors, I. Skog, and P. Händel "Foot-mounted Inertial Navigation and Cooperative Sensor Fusion for Indoor Positioning," Proceedings of ION International Technical Meeting (ITM), San Diego, USA, January 2010.

[9] J. Rydell and E. Emilsson, "CHAMELEON: Visual-inertial indoor navigation," Proceedings of Position Location and Navigation Symposium (PLANS), Myrtle Beach, SC, USA, April 2012.

[10] J. Bird and D. Arden, "Indoor navigation with foot-mounted strapdown inertial navigation and magnetic sensors," IEEE Wireless Communications Magazine, April 2011. 
